\title{
Effects of heart rate and atropine on 'dual AV conduction'
}

\author{
H. Neuss, M. Schlepper, and H. F. Spies, with the technical assistance of Ch. De Mey \\ From the Kerckhoff-Klinik, Bad Nauheim, West Germany
}

In patients with evidence of dual $A V$ conduction, a premature atrial depolarization is normally conducted via a fast conducting pathway with a long refractory period. At a critically timed coupling interval, the effective refractory period is reached and now the impulse is conducted over a pathway with a slow conduction velocity and a short effective refractory period. At this moment in the His bundle electrocardiogram, a sudden increase in the AH interval occurs which is called the 'break' phenomenon.

This phenomenon was studied in 14 patients, with a history of supraventricular paroxysmal tachycardia, at the patient's own heart rate, at different paced heart rates, and after the administration of atropine. In 11 patients the 'break' phenomenon could be elicited during sinus rhythm; in 3 when the heart rate was accelerated. The effective refractory period of the fast conducting pathway was prolonged in 9 patients, remained unchanged in 3, and was shortened in 2 when the heart rate was increased. The effective refractory period of the slow conducting pathway was reached when block occurred proximal to the His bundle. This parameter was increased in 8 patients, remained unchanged in 3, and was decreased in 1 patient with augmentation of the heart rate. This effective refractory period was not measured in 2 patients. Atropine led to a shortening of the refractory period of both pathways in 8 patients studied. Atrial echo phenomena indicating the beginning of a re-entry circuit occurred at an AH prolongation that was not always identical to that measured at the point of 'break'. Widening, as well as narrowing, of the echo zone was found, indicating that the re-entry circuit was in a state of labile equilibrium. In cases where shortening of the pathways involved in the re-entry circuit was found, structures other than nodal may exist; therefore paranodal bypass must be considered.

Animal experiments (Janse et al., 1971; Van Capelle et al., 1972; Moe, Cohen, and Vick, 1963) as well as electrophysiological studies in the intact human heart (Goldreyer and Bigger, 1971; Goldreyer, Weiss, and Damato, 1971) suggest that the existence of two parallel conducting pathways between atria and ventricles is responsible for the occurrence of atrial and ventricular echoes and for certain types of supraventricular tachycardias. Their presence is thought to be caused by longitudinal dissociation of the AV junction rather than to different morphological structures. 'Whether or not 2 anatomically distinct pathways exist, they cannot serve as the substrate for reciprocal responses unless they also differ functionally. They may differ with respect to refractory period duration, margin of safety, response to adrenergic or cholinergic neural influences, response to drugs Received 5 June 1975. or ionic environment, or any combination of these; but differ they must' (Moe and Mendez, 1966).

To evaluate the functional differences in the two pathways between atria and the bundle of His in the intact human heart it is necessary that both pathways differ not only in respect to their refractory behaviour but in regard to their conduction velocity as well. Functional behaviour of both pathways can be studied, provided the effective refractory period of the faster conducting pathway is longer than the effective refractory period of the slower conducting pathway. Under these circumstances a sudden prolongation of the $\mathrm{AH}$ interval induced by premature atrial depolarizations occurs when a critical shortening of the coupling interval is reached (Denes et al., 1973; Denes, Wu, and Rosen, 1974b; Rosen, Mehta, and Miller, 1974). The sudden 'break' in AV nodal conduction time allows the measurement of refractory periods of 
both pathways between atria and the bundle of His, as suggested by Denes et al. (1974b).

This break phenomenon will occur when the faster conducting pathways become refractory and thus the precondition for triggering paroxysmal supraventricular tachycardia (PSVT) is established. It is, however, not always possible to elicit a break phenomenon at the patient's own basic heart rate. We therefore studied the influence of a variable heart rate and atropine administration on the break phenomenon and the trigger mechanism for atrial echoes and supraventricular tachycardia in 14 patients with a history of paroxysmal supraventricular tachycardia.

\section{Patients and methods}

Fourteen patients ( 6 male and 8 female; ages ranging from 17 to 56 years) with paroxysmal supraventricular tachycardia who exhibited a break of $\mathrm{AH}$ interval during premature atrial stimulation were studied. None of the patients showed electrocardiographic patterns of WPW syndrome. In 5 patients $P R$ intervals were $130 \mathrm{~ms}$ or less (see Table 1).

All patients consented to the electrophysiological study and were in a non-sedated state and without antiarrhythmic medication at the time of examination. A 5-electrode catheter was placed via the right femoral vein at the right side of the ventricular septum adjacent to the bundle of His according to Scherlag et al. (1969). Two appropriate electrodes were selected to record filtered $(40-200 \mathrm{~Hz})$ low right atrial potentials and bundle of His depolarizations (HBE). A second catheter with four electrodes was positioned via the same femoral vein in the proximity of the sinus node. Two electrodes were used to record high right atrial potentials (AE), while the remaining two electrodes served for atrial stimulation. Simultaneously, several surface electrocardiographic leads were registered. Surface electrocardiographic and intracardiac electrograms were recorded on magnetic tape and displayed by means of an ink writing system with a paper speed of $100 \mathrm{~mm} / \mathrm{s}$. The atrial stimuli consisted of rectangular impulses of $2.0 \mathrm{~ms}$ duration and twice diastolic threshold.

As a first step the driving cycle length was gradually decreased by steps of $10 \mathrm{~ms}$ to a minimal SS interval of $300 \mathrm{~ms}$, and the frequency-dependent prolongation of the intranodal conduction time (AH interval) as well as the appearance of second degree AV block was observed. Secondly, during sinus rhythm and at different paced heart rates refractory periods of the AV conduction system were determined by the extrastimulus method according to Wit et al. (1970). The extrastimulus $\left(\mathrm{S}_{2}\right)$ was introduced after every 8 th driven $\left(S_{1}\right)$ or spontaneous beat. Coupling intervals were decreased by $10 \mathrm{~ms}$ decrements in successive test cycles. PQ, AH, and HQ intervals were measured as previously described (Neuss and Schlepper, 1974). $A_{1}$ and $H_{1}$ represent the atrial and $\mathrm{His}$ bundle potentials of the last beat, whether spontaneous or driven; $A_{2}$ and $\mathrm{H}_{2}$ represent the atrial and $H$ is bundle deflection in response to the extra stimulus.

The effective refractory period of the atrium (ERP A) was derived from the longest $S_{1}-S_{2}$ interval at which atrial capture failed to occur. In some patients appearance of multiple atrial responses to $S_{2}$ prevented the determination of the atrial effective refractory period, as we tried to avoid producing atrial fibrillation by further decreasing $S_{1}-S_{2}$ intervals. The effective refractory period of the fast conducting pathway (ERP FP) was defined as the $A_{1}-A_{2}$ interval at which a sudden prolongation of the $A_{2}-H_{2}$ interval suggested block of the fast conducting pathway. The effective refractory period of the slow conducting pathway (ERP SP) was defined as the longest $A_{1}-A_{2}$ interval at which additional impulse propagation via the slow pathway was blocked. The functional refractory period of the atrium (FRP A) was defined as the shortest possible $A_{1}-A_{2}$ interval which could be elicited by shortening the coupling interval. In 8 patients $0.5 \mathrm{mg}$ atropine sulphate was administered intravenously and the studies were repeated 5 to 10 minutes after the injection.

\section{Results}

Analysis of AV conduction showed $\mathrm{PA}, \mathrm{AH}$, and HQ intervals during sinus rhythm within the normal range in all patients (see Table 1 ).

(1) Atrial pacing with increasing heart rate led to second degree AV block (type I) proximal to the bundle of $\mathrm{His}$ in 10 patients, while $1: 1 \mathrm{AV}$ conduction persisted down to a driving cycle length of $300 \mathrm{~ms}$ in 4 patients.

In one patient (Case 1) second degree AV block occurred early, at a driving cycle length of $700 \mathrm{~ms}$. In this patient an atypical AV nodal Wenckebach phenomenon was observed. The sudden prolongation of the $\mathrm{AH}$ interval (increment $230 \mathrm{~ms}$, from AH 120 to $350 \mathrm{~ms}$ ) at a driving cycle length of $700 \mathrm{~ms}$ resulted in an atrial echo, the AV conduction of which was blocked proximal to the bundle of His. The findings were identical to those presented by Denes et al. (1973). In five other patients (Cases 3, $5,6,9$, and 11 ) a sudden increase of the $\mathrm{AH}$ interval (increment: 100 to $230 \mathrm{~ms}$ ) was observed at a critical driving cycle length. In four patients this 'break' in the $\mathrm{AH}$ interval initiated a paroxysmal supraventricular tachycardia as demonstrated in Fig. 1 (Case 11). When in this patient the atrial pacing cycle length was decreased from 330 to $320 \mathrm{~ms}$ a sudden prolongation of the $\mathrm{AH}$ interval (140 to $230 \mathrm{~ms}$ ) occurred. Supraventricular tachycardia became evident when pacing was discontinued.

(2) Refractory periods: The refractory periods of the atrium and the AV node (AVN) observed at different heart rates are summarized in Table 2. 
TABLE 1

\begin{tabular}{|c|c|c|c|c|c|c|c|c|c|c|c|}
\hline $\begin{array}{l}\text { Case } \\
\text { No. }\end{array}$ & $\begin{array}{l}\text { Age } \\
\text { and } \\
\text { sex }\end{array}$ & & $D$ & $\begin{array}{l}\text { Basic } \\
\text { cycle } \\
\text { length } \\
(m s)\end{array}$ & $\begin{array}{l}P A \\
(m s)\end{array}$ & $\begin{array}{l}A H \\
(m s)\end{array}$ & $\begin{array}{l}H Q \\
(m s)\end{array}$ & $\begin{array}{l}\text { Second degree } \\
A V \text { block } \\
S S \text { interval } \\
\text { (ms) }\end{array}$ & $\begin{array}{l}\text { Break of } \\
\text { critical } \\
\text { cycle } \\
\text { length } \\
\text { (ms) }\end{array}$ & $\begin{array}{l}A H \text { at } \\
\text { increasing } \\
A H \text { to } A H \\
(m s)\end{array}$ & $\begin{array}{l}\text { AP rate } \\
\text { echo phen. } \\
\text { (ms) }\end{array}$ \\
\hline 1 & 39 & $\mathbf{M}$ & NM & 1000 & 40 & 70 & 35 & 700 & 700 & $120-350$ & $\mathbf{A}^{\prime}$ \\
\hline 2 & 51 & $\mathbf{M}$ & $\phi$ & 720 & 30 & 120 & 50 & 340 & - & - $\quad-$ & 二 \\
\hline 3 & 17 & F & $\phi$ & 690 & 25 & 90 & 40 & 350 & 410 & $180-280$ & SVT \\
\hline 4 & 54 & F & $\phi$ & 780 & 40 & 100 & 40 & 400 & - & - $\quad-$ & - \\
\hline 5 & 52 & $\mathbf{F}$ & $\phi$ & 810 & 30 & 50 & 50 & - & 320 & $160-280$ & SVT \\
\hline 6 & 61 & $\mathbf{M}$ & CHD & 1030 & 20 & 100 & 50 & 390 & 570 & $160-240$ & - \\
\hline 7 & 30 & $\mathbf{F}$ & $\phi$ & 740 & 30 & 80 & 55 & 330 & - & $-\quad-$ & - \\
\hline 8 & 42 & $\mathbf{M}$ & $\phi$ & 780 & 45 & 50 & 40 & 一 $^{\star}$ & 二 & - - & - \\
\hline 9 & 24 & $\mathbf{M}$ & $\phi$ & 970 & 35 & 95 & 50 & 470 & 500 & $150-360$ & SVT \\
\hline 10 & 32 & F & $\phi$ & 590 & 20 & 60 & 45 & -* & - & $-\quad-$ & - \\
\hline 11 & 23 & $\mathbf{F}$ & $\phi$ & 620 & 30 & 60 & 40 & 一 & 320 & $140-230$ & SVT \\
\hline 12 & 56 & F & $\phi$ & 850 & 20 & 60 & 40 & 380 & - & - - & 一 \\
\hline 13 & 47 & $\mathbf{M}$ & $\phi$ & 780 & 30 & 90 & 50 & 330 & - & - & - \\
\hline 14 & 20 & $\mathbf{F}$ & $\phi$ & 670 & 25 & 55 & 50 & 370 & - & - & - \\
\hline
\end{tabular}

Abbreviations: $\mathrm{D}=$ diagnosis; $\phi=$ no detectable heart disease; $\mathrm{NM}=$ non-acute myocarditis; $\mathrm{CHD}=$ coronary heart disease; $\mathbf{M}=$ male; $\mathrm{F}=$ female; $\mathrm{AP}=$ atrial pacing; $\mathbf{A}^{\prime}=$ atrial echo; $S \mathrm{ST}=$ supraventricular tachycardia.

$\star 1: 1 \mathrm{AV}$ conduction down to a cycle length of $300 \mathrm{~ms}$.

TABLE 2

\begin{tabular}{|c|c|c|c|c|c|c|c|c|c|c|c|c|}
\hline $\begin{array}{l}\text { Case } \\
\text { No. }\end{array}$ & & $\begin{array}{l}\text { Cycle } \\
\text { length } \\
\text { (ms) }\end{array}$ & $\begin{array}{l}E R P F P \\
(m s)\end{array}$ & $\begin{array}{l}F R P S P \\
(m s)\end{array}$ & $\begin{array}{l}E R P A \\
(m s)\end{array}$ & $\begin{array}{l}\text { AH } \\
\text { basic } \\
\text { cycle } \\
\text { length } \\
\text { (ms) }\end{array}$ & $\begin{array}{l}\text { AH break } \\
\text { (ms) }\end{array}$ & $\begin{array}{l}A H \max . \\
(m s)\end{array}$ & Echo. & $\begin{array}{l}\text { Crit. AH } \\
\text { (ms) }\end{array}$ & $\begin{array}{l}R R \\
S V T \\
(m s)\end{array}$ & $\begin{array}{l}H A^{\prime} \\
(m s)\end{array}$ \\
\hline 1 & $\begin{array}{l}\text { C } \\
\text { C } \\
\text { A } \\
\text { A } \\
\text { A }\end{array}$ & $\begin{array}{r}1000 \\
800 \\
800 \\
700 \\
600\end{array}$ & $\begin{array}{c}400 \\
560 \\
<320^{\star} \\
<320^{\star} \\
330\end{array}$ & $\begin{array}{c}380 \\
\geqq 560 \star \star \\
-\star \star \\
-\star \star \star \\
\geqq 330 \star \star\end{array}$ & $\begin{array}{l}320 \\
300 \\
300 \\
300 \\
-\star \star\end{array}$ & $\begin{array}{r}70 \\
100 \\
75 \\
80 \\
90\end{array}$ & $\begin{array}{l}160-450 \\
-\quad- \\
-\quad- \\
-\quad-\end{array}$ & $\begin{array}{l}460 \\
150 \\
120 \\
150 \\
130\end{array}$ & $\begin{array}{l}\mathbf{A}^{\prime} \\
- \\
-\end{array}$ & $\begin{array}{l}450 \\
- \\
- \\
-\end{array}$ & $\begin{array}{l}- \\
z \\
-\end{array}$ & $\begin{array}{l}190 \\
- \\
- \\
-\end{array}$ \\
\hline 2 & $\begin{array}{l}\text { C } \\
\text { C } \\
\text { C } \\
\text { C } \\
\text { A } \\
\text { A } \\
\text { A } \\
\text { A }\end{array}$ & $\begin{array}{l}720 \\
600 \\
500 \\
400 \\
700 \\
600 \\
500 \\
400\end{array}$ & $\begin{array}{c}<290^{\star} \\
310 \\
310 \\
310 \\
280 \\
250 \\
260 \\
290\end{array}$ & $\begin{aligned} & \overline{2}^{\star \star} \\
&<250^{\star}<250^{\star} \\
& 280 \\
& \geqq 290^{\star \star} \geqq 250^{\star \star} \\
& \geqq 260 \star \star \\
& 250\end{aligned}$ & 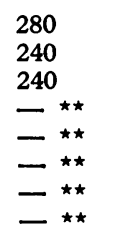 & $\begin{array}{l}120 \\
120 \\
180 \\
230 \\
120 \\
120 \\
140 \\
190\end{array}$ & $\begin{array}{l}\overline{270}-\overline{330} \\
260-310 \\
280-340 \\
=- \\
\overline{-}- \\
270-340\end{array}$ & $\begin{array}{l}210 \\
370 \\
410 \\
470 \\
210 \\
210 \\
280 \\
400\end{array}$ & $\begin{array}{l}\overline{-} \\
\overline{\text { svT }} \\
\overline{-} \\
\overline{\text { sVT }}\end{array}$ & $\begin{array}{l}- \\
\overline{-} \\
430 \\
- \\
\overline{400}\end{array}$ & $\begin{array}{l}- \\
\overline{-} \\
400 \\
\overline{-} \\
\overline{370}\end{array}$ & $\begin{array}{l}\bar{z} \\
\overline{100} \\
\overline{-} \\
\overline{100}\end{array}$ \\
\hline 3 & $\begin{array}{l}\mathrm{C} \\
\mathrm{C}\end{array}$ & $\begin{array}{l}690 \\
500\end{array}$ & $\begin{array}{l}350 \\
390\end{array}$ & $\begin{array}{l}280 \\
280\end{array}$ & $\bar{Z}^{\star \star \star}$ & $\begin{array}{l}90 \\
90\end{array}$ & $\begin{array}{l}160-270 \\
160-260\end{array}$ & $\begin{array}{l}330 \\
340\end{array}$ & $\begin{array}{l}\text { SVT } \\
\text { SVT }\end{array}$ & $\begin{array}{l}270 \\
320\end{array}$ & $\begin{array}{l}380 \\
380\end{array}$ & $\begin{array}{l}65 \\
65\end{array}$ \\
\hline 4 & $\begin{array}{l}\text { C } \\
\text { C } \\
\text { C }\end{array}$ & $\begin{array}{l}780 \\
600 \\
500\end{array}$ & $\begin{array}{l}330 \\
330 \\
350\end{array}$ & $\begin{array}{c}<320 \star \star \\
280 \\
300\end{array}$ & $\begin{array}{l}Z^{\star \star \star} \\
\text { - }^{\star \star \star}\end{array}$ & $\begin{array}{l}100 \\
170 \\
200\end{array}$ & $\begin{array}{l}260-480 \\
230-450 \\
240-410\end{array}$ & $\begin{array}{c}480 \\
490 \\
480\end{array}$ & $\begin{array}{l}\text { SVT } \\
\text { SVT } \\
\text { SVT }\end{array}$ & $\begin{array}{l}480 \\
470 \\
410\end{array}$ & $\begin{array}{l}510 \\
510 \\
510\end{array}$ & $\begin{array}{l}120 \\
120 \\
120\end{array}$ \\
\hline 5 & $\begin{array}{l}\text { C } \\
\text { C } \\
\text { C } \\
\text { C } \\
\text { C } \\
\text { A } \\
\text { A }\end{array}$ & $\begin{array}{l}810 \\
700 \\
600 \\
500 \\
400 \\
500 \\
400\end{array}$ & $\begin{array}{c}<290^{\star} \\
290 \\
320 \\
310 \\
310 \\
<230^{\star} \\
<220^{\star}\end{array}$ & $\begin{array}{l}\bar{Z}^{\star \star \star} \\
<260^{\star} \\
<230^{\star} \\
250 \\
<310^{\star \star} \\
-\star \star \star \\
-\star \star\end{array}$ & $\begin{array}{l}270 \\
230 \\
210 \\
200 \\
\overline{2}_{200} \\
200\end{array}$ & $\begin{array}{r}50 \\
70 \\
70 \\
90 \\
110 \\
70 \\
80\end{array}$ & $\begin{array}{l}\overline{180}-240 \\
120-260 \\
130-250 \\
150-250 \\
--\overline{-} \\
--\end{array}$ & $\begin{array}{r}90 \\
270 \\
320 \\
290 \\
250 \\
160 \\
160\end{array}$ & $\begin{array}{l}-\overline{A^{\prime}} \\
\text { SVT } \\
\text { SVT } \\
\text { A' }^{\prime} \\
- \\
-\end{array}$ & $\begin{array}{l}- \\
240 \\
270 \\
270 \\
250 \\
- \\
-\end{array}$ & $\begin{array}{l}- \\
\overline{330} \\
330 \\
- \\
-\end{array}$ & $\begin{array}{l}- \\
90 \\
90 \\
90 \\
90 \\
-\end{array}$ \\
\hline
\end{tabular}


TABLE 2 Continued

\begin{tabular}{|c|c|c|c|c|c|c|c|c|c|c|c|c|}
\hline $\begin{array}{l}\text { Case } \\
\text { No. }\end{array}$ & & $\begin{array}{l}\text { Cycle } \\
\text { length } \\
\text { (ms) }\end{array}$ & $\begin{array}{l}E R P F P \\
(m s)\end{array}$ & $\begin{array}{l}E R P S P \\
(m s)\end{array}$ & $\begin{array}{l}E R P A \\
\text { (ms) }\end{array}$ & $\begin{array}{l}\text { AH } \\
\text { basic } \\
\text { cycle } \\
\text { length } \\
\text { (ms) }\end{array}$ & $\begin{array}{l}\text { AH break } \\
\text { (ms) }\end{array}$ & $\begin{array}{l}A H \text { max. } \\
\text { (ms) }\end{array}$ & Echo. & $\begin{array}{l}\text { Crit. } A H \\
\text { (ms) }\end{array}$ & $\begin{array}{l}R R \\
S V T \\
(m s)\end{array}$ & $\begin{array}{l}H A^{\prime} \\
\text { (ms) }\end{array}$ \\
\hline 6 & $\begin{array}{l}\text { C } \\
\text { C } \\
\text { C } \\
\text { A } \\
\text { A }\end{array}$ & $\begin{array}{r}1030 \\
600 \\
500 \\
900 \\
500\end{array}$ & $\begin{array}{l}460 \\
\frac{\bar{C}^{\star \star}}{340} \\
390\end{array}$ & $\begin{array}{c}<330 \star \\
330 \\
320 \\
290 \\
290\end{array}$ & $\begin{array}{l}310 \\
280 \\
270 \\
\overline{250}^{\star \star \star}\end{array}$ & $\begin{array}{r}100 \\
150 \\
280 \\
80 \\
130\end{array}$ & $\begin{array}{l}130-220 \\
\overline{1}-\overline{-} \\
160-210 \\
160-240\end{array}$ & $\begin{array}{l}320 \\
330 \\
350 \\
240 \\
340\end{array}$ & $\begin{array}{l}\mathbf{A}^{\prime} \\
\mathbf{A}^{\prime} \\
\mathbf{A}^{\prime} \\
\overline{\mathbf{A}^{\prime}}\end{array}$ & $\begin{array}{l}300 \\
290 \\
320 \\
\frac{-}{290}\end{array}$ & $\begin{array}{l}- \\
z \\
-\end{array}$ & $\begin{array}{l}90 \\
90 \\
90 \\
- \\
90\end{array}$ \\
\hline 7 & $\begin{array}{l}\text { C } \\
\text { C } \\
\text { C } \\
\text { A } \\
\text { A }\end{array}$ & $\begin{array}{l}740 \\
600 \\
400 \\
520 \\
400\end{array}$ & $\begin{aligned}< & 280^{\star} \\
& 270 \\
& 290 \\
< & 230 \star \star \\
< & 220 \star \star\end{aligned}$ & $\begin{array}{l}\bar{D}^{\star \star \star} \\
<260^{\star} \\
<270^{\star \star} \\
-\star \star \\
-\star \star\end{array}$ & $\begin{array}{l}240 \\
220 \\
\bar{Z}^{\star \star} \\
210\end{array}$ & $\begin{array}{r}80 \\
85 \\
220 \\
80 \\
90\end{array}$ & $\begin{array}{l}\overline{280}-\overline{450} \\
300-370 \\
-\quad- \\
--\end{array}$ & $\begin{array}{l}250 \\
470 \\
370 \\
180 \\
220\end{array}$ & $\begin{array}{l}- \\
z \\
-\end{array}$ & $\begin{array}{l}- \\
z \\
-\end{array}$ & $\begin{array}{l}- \\
z \\
-\end{array}$ & $\begin{array}{l}\bar{z} \\
\bar{z}\end{array}$ \\
\hline 8 & $\begin{array}{l}C \\
C \\
C \\
C \\
C\end{array}$ & $\begin{array}{l}780 \\
600 \\
500 \\
400 \\
300\end{array}$ & $\begin{array}{l}310 \\
300 \\
260 \\
250 \\
210\end{array}$ & $\begin{array}{c}<270 \star \\
230 \\
230 \\
230 \\
200\end{array}$ & $\begin{array}{l}260 \\
200 \\
\frac{1}{190}^{\star \star} \\
-^{\star \star}\end{array}$ & $\begin{array}{l}50 \\
60 \\
80 \\
75 \\
90\end{array}$ & $\begin{array}{l}100-190 \\
100-170 \\
140-210 \\
120-190 \\
120-220\end{array}$ & $\begin{array}{l}200 \\
220 \\
220 \\
210 \\
220\end{array}$ & $\begin{array}{l}A^{\prime} \\
\text { SVT } \\
\text { SVT } \\
\text { SVT } \\
\text { A }^{\prime}\end{array}$ & $\begin{array}{l}190 \\
170 \\
210 \\
190 \\
220\end{array}$ & $\begin{array}{l}- \\
320 \\
260 \\
270 \\
-\end{array}$ & $\begin{array}{l}110 \\
110 \\
110 \\
110 \\
110\end{array}$ \\
\hline 9 & $\begin{array}{l}\mathrm{C} \\
\mathrm{C}\end{array}$ & $\begin{array}{l}970 \\
600\end{array}$ & $\begin{array}{l}410 \\
440\end{array}$ & $\begin{array}{l}380 \\
340\end{array}$ & $\begin{array}{l}290 \\
260\end{array}$ & $\begin{array}{r}95 \\
160\end{array}$ & $\begin{array}{l}290-360 \\
240-320\end{array}$ & $\begin{array}{l}370 \\
430\end{array}$ & $\begin{array}{l}\text { A' }^{\prime} \\
\text { SVT }\end{array}$ & $\begin{array}{l}360 \\
320\end{array}$ & $\overline{450}$ & $\begin{array}{l}20 \\
20\end{array}$ \\
\hline 10 & $\begin{array}{l}\mathrm{C} \\
\mathrm{C}\end{array}$ & $\begin{array}{l}500 \\
400\end{array}$ & $\begin{array}{l}300 \\
300\end{array}$ & $\begin{array}{l}<270^{\star \star \star} \\
<260^{\star \star}\end{array}$ & $Z^{\star \star \star}$ & $\begin{array}{r}60 \\
110\end{array}$ & $\begin{array}{l}160-200 \\
120-180\end{array}$ & $\begin{array}{l}220 \\
220\end{array}$ & $\begin{array}{l}\text { SVT } \\
\text { SVT }\end{array}$ & $\begin{array}{l}220 \\
200\end{array}$ & $\begin{array}{l}280 \\
290\end{array}$ & $\begin{array}{l}90 \\
90\end{array}$ \\
\hline 11 & $\begin{array}{l}\mathrm{C} \\
\mathrm{C}\end{array}$ & $\begin{array}{l}620 \\
400\end{array}$ & $\begin{array}{l}320 \\
260\end{array}$ & $\begin{array}{l}<300 \star \star \\
<260 \star \star\end{array}$ & $\bar{Z}^{\star \star \star}$ & $\begin{array}{l}60 \\
80\end{array}$ & $\begin{array}{l}100-220 \\
140-240\end{array}$ & $\begin{array}{l}220 \\
240\end{array}$ & $\begin{array}{l}\text { SVT } \\
\text { SVT }\end{array}$ & $\begin{array}{l}220 \\
240\end{array}$ & $\begin{array}{l}300 \\
300\end{array}$ & $\begin{array}{l}90 \\
90\end{array}$ \\
\hline 12 & $\begin{array}{l}\text { C } \\
\text { C } \\
\text { C } \\
\text { C } \\
\text { A } \\
\text { A } \\
\text { A } \\
\text { A }\end{array}$ & $\begin{array}{l}850 \\
700 \\
600 \\
500 \\
700 \\
600 \\
500 \\
400\end{array}$ & $\begin{array}{c}340 \\
330 \\
330 \\
350 \\
<290 \\
<260 \star \\
290 \\
300\end{array}$ & $\begin{array}{c}300 \\
290 \\
290 \\
290 \\
\bar{Z}^{\star \star \star} \\
\bar{C}^{\star \star} \\
230^{\star} \\
260\end{array}$ & $\begin{array}{l}250 \\
230 \\
230 \\
\overline{2}^{\star 230} \\
190 \\
180 \\
170\end{array}$ & $\begin{array}{l}60 \\
70 \\
80 \\
90 \\
50 \\
70 \\
70 \\
90\end{array}$ & $\begin{array}{l}120-210 \\
150-300 \\
130-420 \\
120-250 \\
-= \\
\overline{130}-200 \\
120-190\end{array}$ & $\begin{array}{l}280 \\
510 \\
480 \\
570 \\
100 \\
150 \\
220 \\
230\end{array}$ & $\begin{array}{l}\text { A' }^{\prime} \\
\text { SVT } \\
\text { A }^{\prime} \\
\text { SVT } \\
= \\
= \\
= \\
-\end{array}$ & $\begin{array}{l}280 \\
300 \\
420 \\
460 \\
= \\
= \\
-\end{array}$ & $\begin{array}{l}\overline{480} \\
\overline{480} \\
= \\
= \\
=\end{array}$ & $\begin{array}{l}80 \\
80 \\
80 \\
80 \\
- \\
- \\
-\end{array}$ \\
\hline 13 & $\begin{array}{l}\text { C } \\
\text { C } \\
\text { C } \\
\text { A } \\
\text { A } \\
\text { A }\end{array}$ & $\begin{array}{l}780 \\
600 \\
500 \\
600 \\
500 \\
400\end{array}$ & $\begin{array}{c}320 \\
350 \\
350 \\
<250^{\star} \\
<240^{\star} \\
<230^{\star}\end{array}$ & $\begin{array}{l}280 \\
330 \\
Z^{\star \star} \\
Z^{\star \star} \\
Z^{\star \star}\end{array}$ & $\begin{array}{l}230 \\
200 \\
190 \\
220 \\
200 \\
190\end{array}$ & $\begin{array}{r}90 \\
110 \\
120 \\
50 \\
80 \\
100\end{array}$ & $\begin{array}{l}180-300 \\
200-310 \\
-= \\
=- \\
--\end{array}$ & $\begin{array}{l}470 \\
380 \\
190 \\
170 \\
180 \\
200\end{array}$ & $\begin{array}{l}\mathrm{A}^{\prime} \\
\mathrm{A}^{\prime} \\
- \\
- \\
-\end{array}$ & $\begin{array}{l}300 \\
310 \\
- \\
- \\
-\end{array}$ & $\begin{array}{l}z \\
z \\
z\end{array}$ & $\begin{array}{l}130 \\
120 \\
= \\
- \\
-\end{array}$ \\
\hline 14 & $\begin{array}{l}\mathbf{C} \\
\mathbf{C} \\
\mathbf{C} \\
\mathbf{A} \\
\mathbf{A}\end{array}$ & $\begin{array}{l}670 \\
500 \\
400 \\
500 \\
400\end{array}$ & $\begin{array}{c}320 \\
320 \\
360 \\
<270^{\star} \\
240\end{array}$ & $\begin{array}{c}<290 \star \\
260 \\
300 \\
\overline{220}^{\star \star}\end{array}$ & $\begin{array}{l}250 \\
180 \\
\frac{240}{2} \\
-\star \star\end{array}$ & $\begin{array}{r}60 \\
70 \\
100 \\
40 \\
65\end{array}$ & $\begin{array}{l}120-300 \\
200-340 \\
120-310 \\
-\frac{140}{1410}\end{array}$ & $\begin{array}{r}330 \\
450 \\
380 \\
80 \\
440\end{array}$ & $\begin{array}{l}\mathbf{A}^{\prime} \\
\mathbf{A}^{\prime} \\
\mathbf{A}^{\prime} \\
\overline{\text { SVT }}\end{array}$ & $\begin{array}{l}300 \\
340 \\
310 \\
-10\end{array}$ & $\begin{array}{l}\bar{z} \\
\overline{3} \\
330\end{array}$ & $\begin{array}{l}35 \\
35 \\
35 \\
- \\
25\end{array}$ \\
\hline
\end{tabular}

Abbreviations: ERP FP=effective refractory period of the fast conducting pathway; ERP SP=effective refractory period of the slow conducting pathway; ERP $A=$ effective refractory period of the atria; $A H$ break= sudden prolongation of $A_{2}-H_{2}$ interval from. . . to ...; AH max.=maximal observed prolongation of $A_{2}-H_{2}$ interval; echo.=echo phenomena; crit. $A H=c r i t i c a l ~ A_{2}-H_{2}$ interval at which echo phenomena appear; RR SVT=cycle length of tachycardia (PSVT); HA ${ }^{\prime}=$ retrograde conduction time for atrial echoes ( $H$ potential to retrograde atrial deflection in $\mathrm{HBE}$ ); $C=$ control; $A=$ after atropine; ${ }^{\star}=E R P F P$ or $S P$ is shorter than the shortest possible $A_{1}-A_{2}$ interval; ${ }^{\star \star}=$ not measured.

In 11 patients a sudden prolongation of the $A_{2}-H_{2}$ interval could be provoked during sinus rhythm, so the effective refractory period of the fast pathway
(ERP FP) could be measured. These sudden prolongations of $\mathrm{A}_{2}-\mathrm{H}_{2}$ ranged from 70 to $270 \mathrm{~ms}$. In the remaining three patients (Cases 2, 5, and 7) 

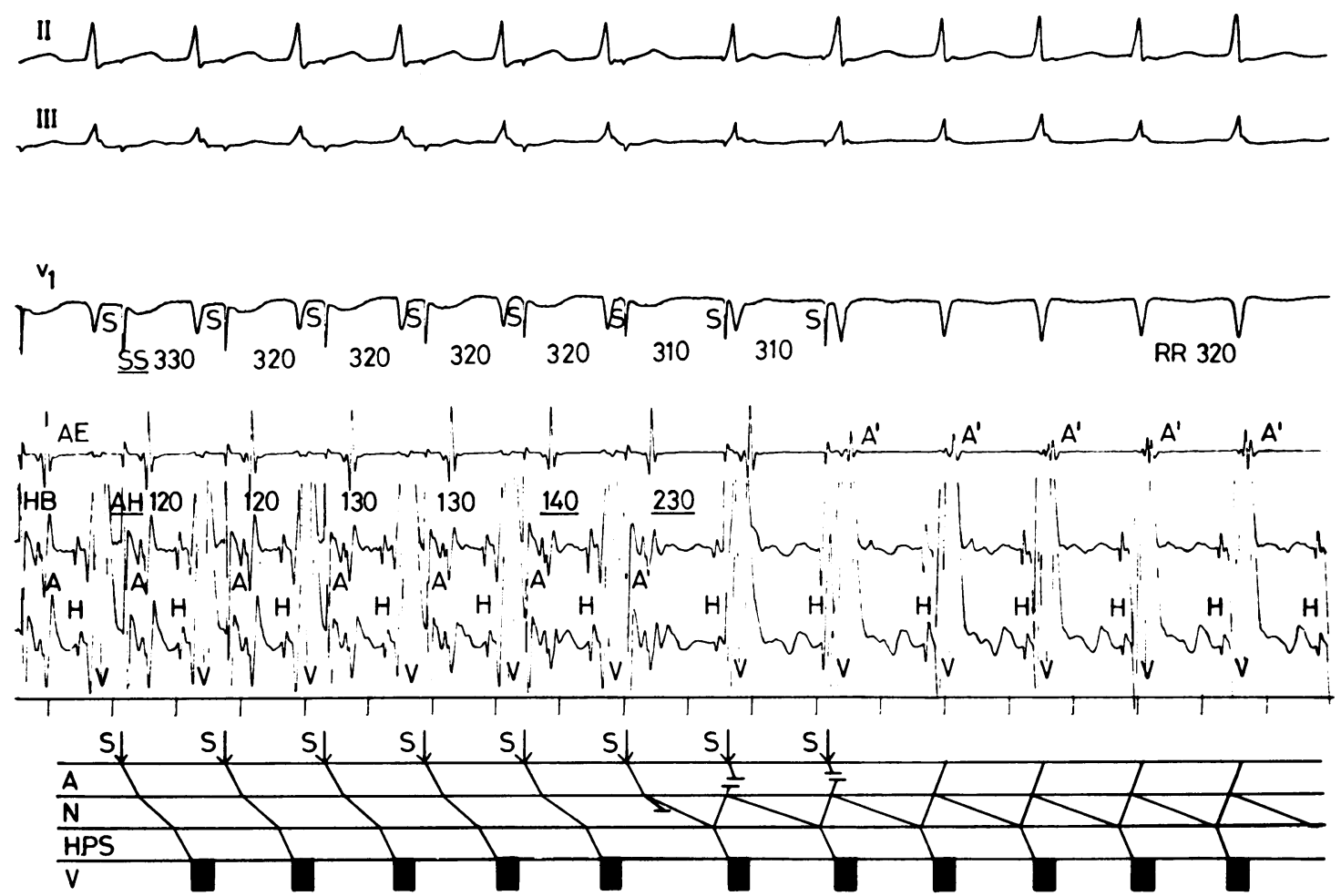

FIG. 1 Electrocardiogram (leads $I I, I I I$, and $V_{1}$ ), high right atrial electrogram ( $A E$ ), and His bundle electrogram $(H B)$ in Case 11. In this and all subsequent figures: $S=$ stimulus artefact, $A=$ atrial potential, $A^{\prime}=$ retrograde atrial depolarization, $H=H i$ bundle potential, $V=$ ventricular potentials. Conventional ladder diagram: $A=$ atrial level, $N=$ level of $A V$ junction, HPS = His-Purkinje system, $V=$ ventricular myocardium.

During atrial pacing with a cycle length of $320 \mathrm{~ms}$ a sudden prolongation of the AH interval from 140 to $230 \mathrm{~ms}$ was observed. When pacing was discontinued a paroxysm of supraventricular tachycardia with a cycle length of $320 \mathrm{~ms}$ became evident. Time marks =200 ms.

no break phenomenon became apparent. At intervals shorter than the ERP FP atrial echoes occurred in all 11 patients, 4 of whom developed a paroxysm of supraventricular tachycardia.

In 5 of the 11 patients showing the break phenomenon the effective refractory period of the slow pathway (ERP SP) could be measured only when further shortening of $A_{1}-A_{2}$ resulted in $A V$ block above the bundle of His. In 3 patients $A V$ conduction via the slow conducting pathway was limited by the effective refractory period of the atria; in the remaining 3 patients the ERP SP could not be measured for technical reasons (multiple response to $A_{2}$ ).

(3) Rate-dependency of refractory periods of the atrioventricular node: In the 3 patients in whom no break phenomenon was apparent at sinus rhythm it could be provoked when basic cycle length was decreased. This was because of a prolongation of the effective refractory period of the fast pathway and/or a shortening of the effective refractory period of the atria.

A rate-dependent prolongation of the effective refractory period of the fast pathway was found in 9 patients. An example is given in Fig. 2 (Case 4): at a basic cycle length of $600 \mathrm{~ms}$ a premature atrial depolarization with a $S_{1}-S_{2}$ interval (identical with an $A_{1}-A_{2}$ interval) of $340 \mathrm{~ms}$ was conducted with an $\mathrm{A}_{2}-\mathrm{H}_{2}$ interval of $220 \mathrm{~ms}$ (Fig. 2a). Shortening of $S_{1}-S_{2}$ to $330 \mathrm{~ms}$ resulted in prolongation of $A_{2}-H_{2}$ to $450 \mathrm{~ms}$. The first atrial echo, which then initiated a paroxysm of supraventricular tachycardia, was elicited at an $A_{1}-A_{2}$ interval of 300 ms (Fig. 2b), the corresponding $\mathrm{A}_{2}-\mathrm{H}_{2}$ interval being $490 \mathrm{~ms}$. At 

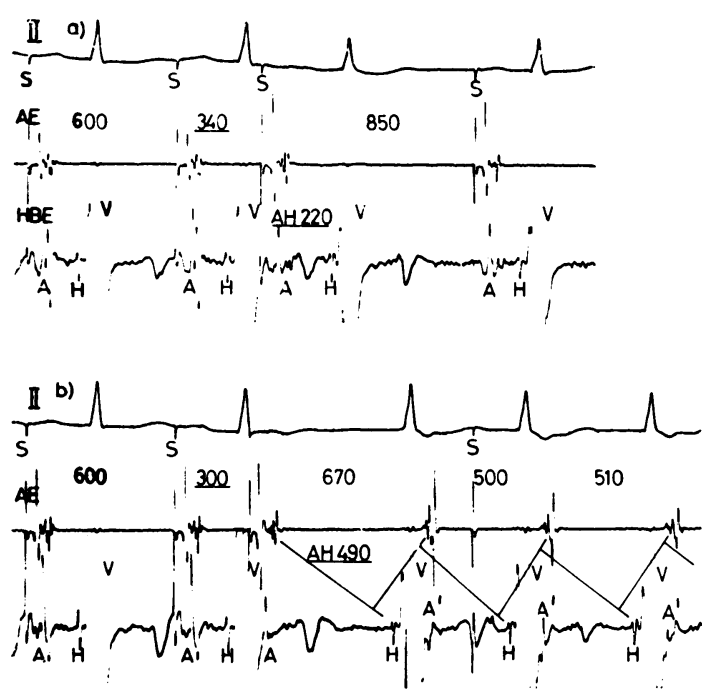
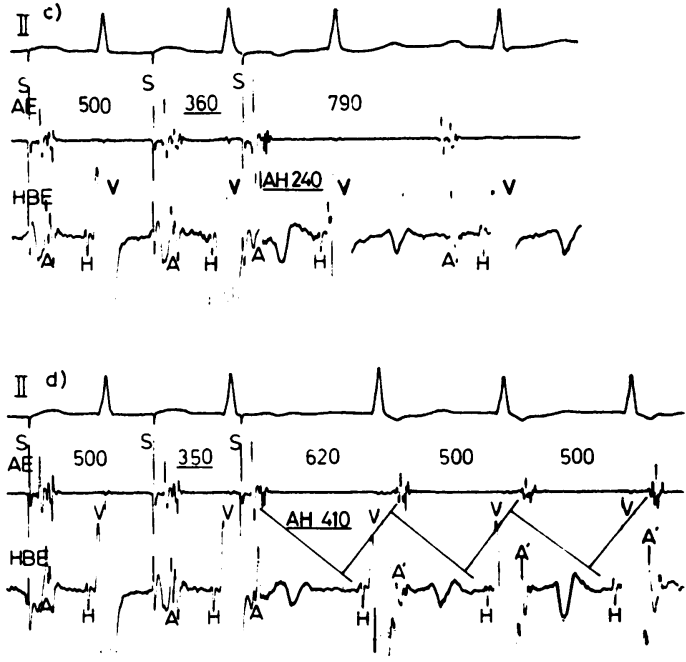

FIG. 2 Electrocardiogram (lead II), atrial electrogram ( $A E$ ), and His bundle recording (HBE) in Case 4. (a) Basic cycle length $600 \mathrm{~ms}$ : a premature atrial depolarization with an $S_{1}-S_{2}$ interval of $340 \mathrm{~ms}$ was conducted with an $A_{2}-H_{2}$ interval of $220 \mathrm{~ms}$. (b) Basic cycle length $600 \mathrm{~ms}$ : at an $S_{1}-S_{2}$ interval of $300 \mathrm{~ms}$ an $A_{2}-H_{2}$ interval of $490 \mathrm{~ms}$ resulted, and atrial echo initiating a paroxysm of supraventricular tachycardia occurred. (c) Basic cycle length $500 \mathrm{~ms}$ : a premature atrial depolarization with an $S_{1}-S_{2}$ interval of 360 ms was conducted with an $\mathrm{A}_{2}-\mathrm{H}_{2}$ interval of $240 \mathrm{~ms}$. (d) Basic cycle length $500 \mathrm{~ms}$ : shortening of $S_{1}-S_{2}$ to $350 \mathrm{~ms}$ resulted in a break phenomenon and the occurrence of supraventricular tachycardia.

a basic cycle length of $500 \mathrm{~ms}$ the break phenomenon occurred at an $A_{1}-A_{2}$ interval of $350 \mathrm{~ms}$ (Fig. 2c, 2d). The essential $A_{2}-H_{2}$ interval for the initiation of supraventricular tachycardia was found to be $410 \mathrm{~ms}$ (Fig. 2d).

In 3 patients no significant change in the effective refractory period of the fast pathway was seen (Cases 6, 10, and 12), while in two patients (Cases 8 and 11) this decreased when basic heart rate was accelerated. This is demonstrated in Fig. 3 (Case 8): at a basic cycle length of $600 \mathrm{~ms}$ the effective refractory period of the fast pathway was reached at an $S_{1}-S_{2}$ interval (identical to $A_{1}-A_{2}$ ) of $300 \mathrm{~ms}$. The sudden prolongation of $\mathrm{A}_{2}-\mathrm{H}_{2}$ was accompanied by the appearance of an atrial echo and the initiation of supraventricular tachycardia (Fig. 3a and b). At a basic cycle length of $500 \mathrm{~ms}$ the effective refractory period of the fast pathway was decreased to $260 \mathrm{~ms}$ (Fig. 3c and d).

Shortening of the basic cycle length resulted in a shortening of the effective refractory period of the slow pathway in only 1 patient (Case 9), while it was prolonged in 8 patients. In 3 patients no significant effects of heart rate on it were found.
In two patients the rate dependency of this refractory period was not measured.

In all patients in whom the effective refractory period of the atria was measured at different heart rates a shortening was observed when the heart rate was increased.

(4) The effects of atropine on the refractory periods: All 8 patients investigated showed a decrease of the effective refractory period of the fast pathway after atropine administration. As far as it could be investigated, the slow conducting pathway behaved similarly.

In 4 patients (Cases 1, 5, 7, and 13) the break phenomenon observed under control conditions disappeared after atropine administration and could not be elicited by shortening the basic cycle length. This effect is shown in Fig. 4 (Case 5): under control conditions at a driving cycle length of $500 \mathrm{~ms}$ and a coupling interval of $310 \mathrm{~ms}$ a sudden increase of the $\mathrm{H}_{1}-\mathrm{H}_{2}$ and $\mathrm{A}_{2}-\mathrm{H}_{2}$ interval was observed (Fig. 4a and b). After atropine with the same driving cycle length the break phenomenon disappeared (Fig. 4c) and the $\mathrm{A}_{2}-\mathrm{H}_{2}$ interval increased continuously (Fig. 4d). 


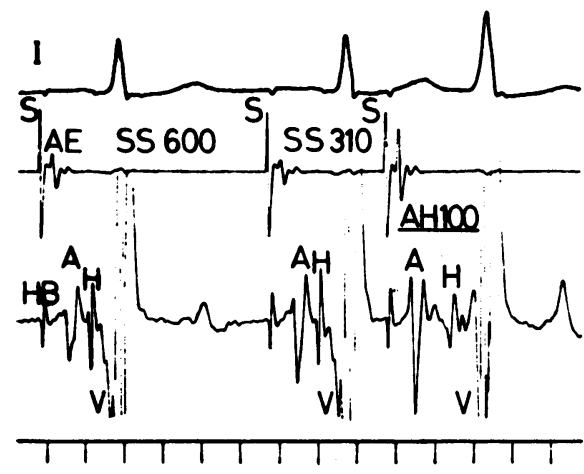

a)

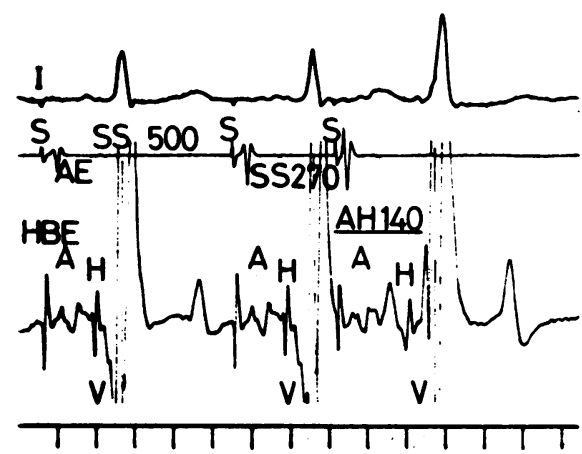

c)

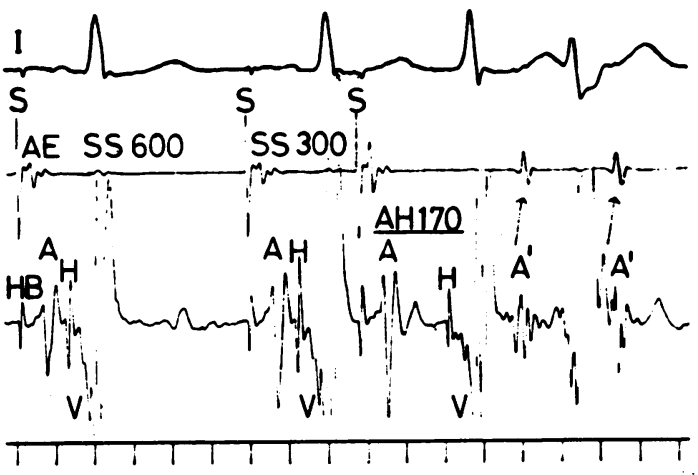

b)

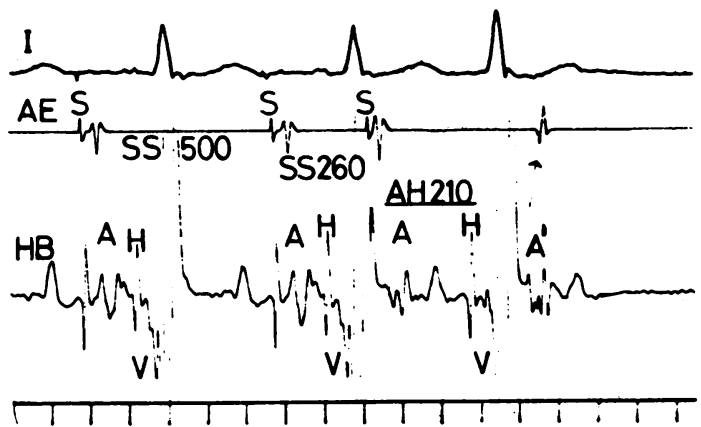

d)

FIG. 3 Electrocardiogram (lead I), atrial electrogram ( $A E$ ), and His bundle recording $(H B E)$ in Case 8. Time marks=100 ms. (a) Basic cycle length $600 \mathrm{~ms}$ : at an $S_{1}-S_{2}$ interval of $310 \mathrm{~ms} \mathrm{~A}_{2}-\mathrm{H}_{2}$ measured $100 \mathrm{~ms}$. (b) Basic cycle length $600 \mathrm{~ms}$ : shortening of $S_{1}-S_{2}$ to 300 ms resulted in a sudden prolongation of $A_{2}-H_{2}$ to $170 \mathrm{~ms}$ and the occurrence of an atrial echo which initiated a supraventricular tachycardia. (c) Basic cycle length 500 ms: at an $S_{1}-S_{2}$ interval of $270 \mathrm{~ms} A_{2}-H_{2}$ measured $140 \mathrm{~ms}$. (d) Basic cycle length $500 \mathrm{~ms}$ : shortening of $S_{1}-S_{2}$ to $260 \mathrm{~ms}$ resulted in a prolongation of $A_{2}-H_{2}$ to $210 \mathrm{~ms}$ and the occurrence of an atrial echo.

In 3 patients at low heart rates after atropine no break phenomenon was observed. However, it could be provoked by shortening the basic cycle length (Cases 8, 12, and 14).

In only one patient could a break phenomenon be detected while in sinus rhythm after atropine. In this case (Case 6: Fig. 5) at a basic cycle length of $500 \mathrm{~ms}$ AV conduction occurred only via the slow conducting pathway under control conditions, as shown by the $A_{2}-H_{2}$ intervals varying between 250 and $280 \mathrm{~ms}$ (Fig. 5a and b). Atrial echoes were seen at a critical $\mathrm{A}_{2}-\mathrm{H}_{2}$ interval of $320 \mathrm{~ms}$ (Fig. 5b). A sudden prolongation of the $\mathbf{A}_{2}-\mathrm{H}_{2}$ interval (from
160 to $240 \mathrm{~ms}$ ) was elicited at an $A_{1}-A_{2}$ interval of $390 \mathrm{~ms}$ after atropine administration. If $\mathrm{A}_{2}-\mathrm{H}_{2}$ was further prolonged to $290 \mathrm{~ms}$ atrial echoes occurred (Fig. 5d). This is graphically demonstrated in Fig. 6. It is evident that atropine (Fig. 6c and d), at basic rate of $120 / \mathrm{min}$, facilitated AV conduction via the fast conducting pathway, so that a break phenomenon could be demonstrated.

(5) The echo phenomena and supraventricular tachycardia: Echo phenomena can be provoked when the AV conduction of a premature atrial depolarization occurs via the slow conducting pathway, while AV conduction down the fast 
FIG. 4 The effects of atropine on the break phenomenon in Case 5 are graphically demonstrated. $\mathrm{H}_{1}-\mathrm{H}_{2}$ intervals (top panel) and $\mathrm{A}_{2}-\mathrm{H}_{2}$ intervals (bottom panel) are depicted as a function of $A_{1}-A_{2}$ intervals. Basic cycle length is $500 \mathrm{~ms}$. In control conditions a sudden prolongation of $\mathrm{H}_{1}-\mathrm{H}_{2}$ (panel a) and $\mathrm{A}_{2}-\mathrm{H}_{2}$ (panel b) occurred at an $A_{1}-A_{2}$ interval of $310 \mathrm{~ms}$. After atropine administration no break phenomenon occurred up to the shortest possible $A_{1}-A_{2}$ interval of $230 \mathrm{~ms}$ (panel $\mathrm{c}$ and $\mathrm{d}$ ).

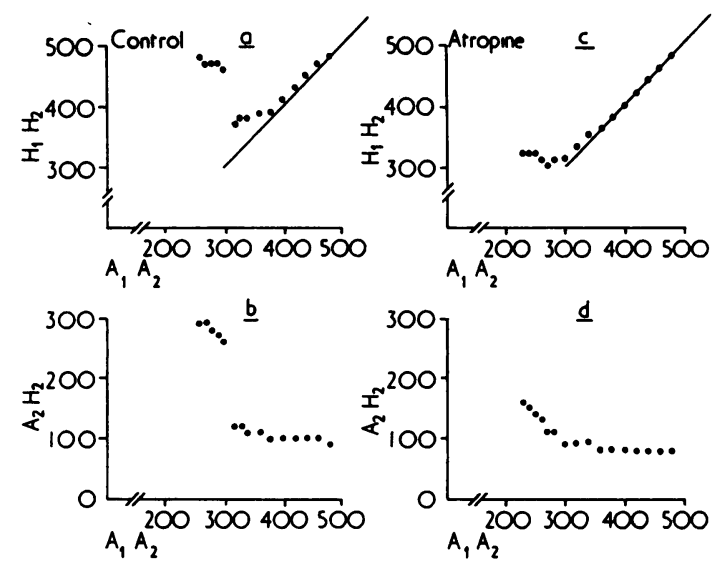

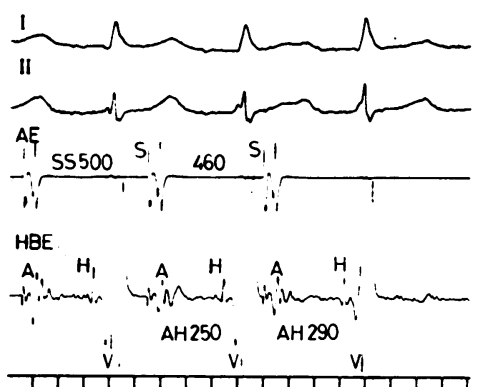

a)
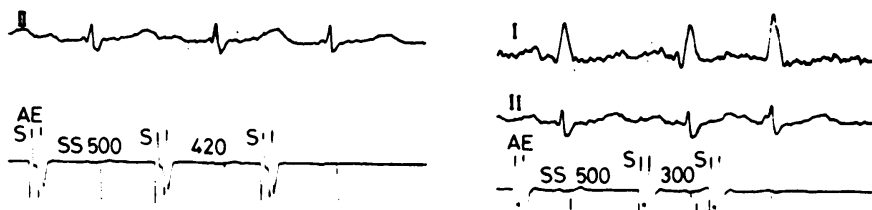

$$
\text { HBE }
$$

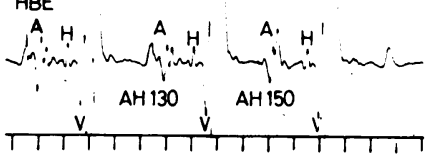

c)

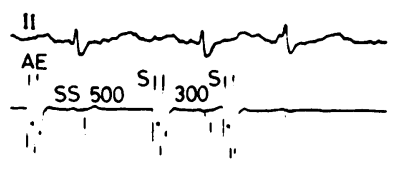

HBE

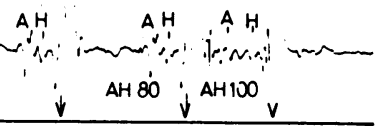

e)
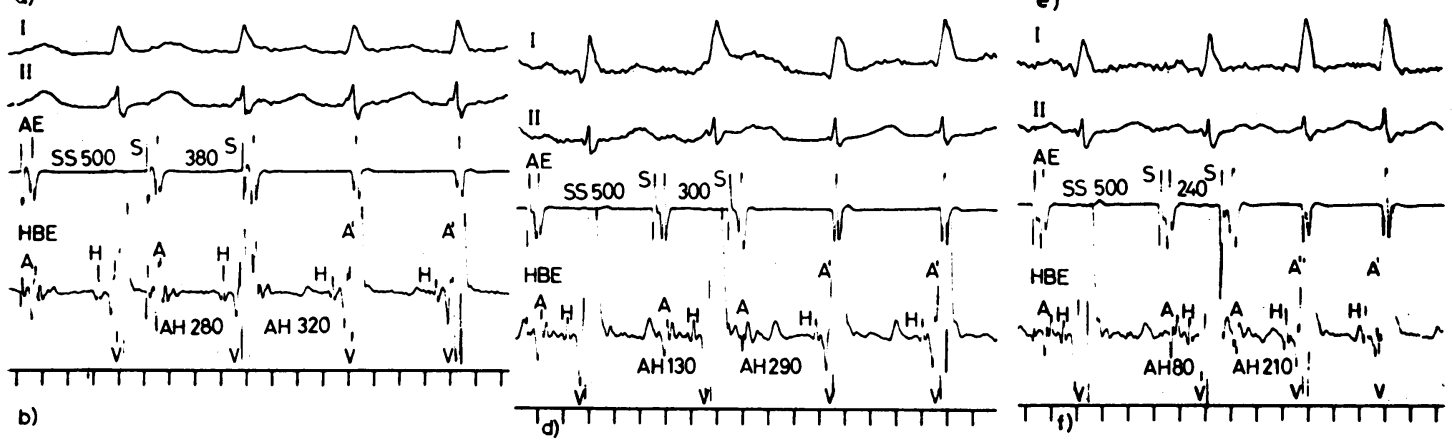

FIG. 5 Electrocardiogram (lead I and II), atrial electrogram ( $A E$ ) and His bundle recording $(H B E)$ in Case 6. Time marks $=100 \mathrm{~ms}$. Basic cycle length $500 \mathrm{~ms}$. Under control conditions $A_{1}-H_{1}$ intervals varied between 250 and $280 \mathrm{~ms}$ (panel a and b). Shortening of $A_{1}-A_{2}$ intervals was accompanied by a smooth increase of $A_{2}-H_{2}$ intervals. At a critical $A_{2}-H_{2}$ interval of $320 \mathrm{~ms}$ (panel b) an atrial echo appeared. After atropine administration (panel $\mathrm{c}$ and d) $A_{1}-H_{1}$ intervals shortened to $130 \mathrm{~ms}$. While a typical break phenomenon occurred at an $A_{1}-A_{2}$ interval of $390 \mathrm{~ms}$ (not shown), the critical $A_{2}-H_{2}$ interval at which atrial echoes appeared was shortened to $290 \mathrm{~ms}$ (panel d). After orciprenaline administration (panel e and f) $A_{1}-H_{1}$ intervals shortened to $80 \mathrm{~ms}$. A typical break phenomenon occurred at an $A_{1}-A_{2}$ interval of $270 \mathrm{~ms}$ (not shown). The critical $A_{2}-H_{2}$ interval was further shortened to $210 \mathrm{~ms}$ (panel f). 


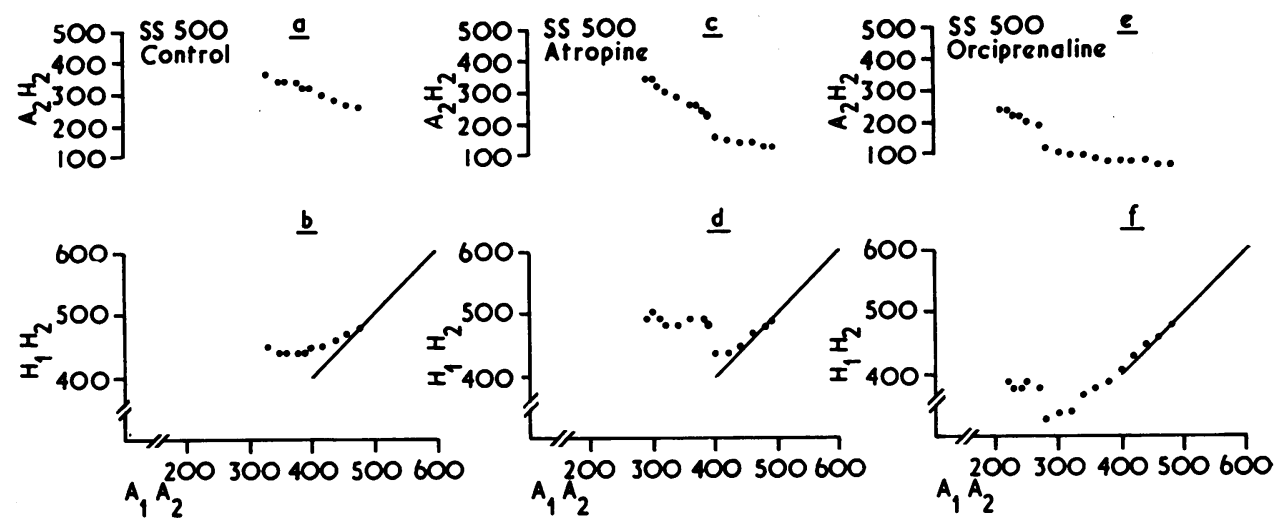

FIG. 6 Graph showing the $A_{2}-H_{2}$ intervals (upper panel) and the $H_{1}-H_{2}$ intervals (lower panel) as a function of the $A_{1}-A_{2}$ intervals. Same case as in Fig. 5. Basic cycle length $500 \mathrm{~ms}$. Under control conditions no break phenomenon was evident (panel a and b). The graph suggests that the appearance of the break phenomenon after atropine administration (panel $\mathrm{c}$ and $\mathrm{d}$ ) and after orciprenaline (panel $\mathrm{e}$ and $\mathrm{f}$ ) is because of improvement of conductivity in a fast-conducting pathway.

conducting pathway is blocked owing to its refractory state. With the exception of Case 6 , this behaviour was demonstrated by the break phenomenon.

Furthermore, the occurrence of an echo phenomenon depends on a critical $\mathrm{A}_{2}-\mathrm{H}_{2}$ interval. In none of the patients could an atrial echo or supraventricular tachycardia be elicited unless $\mathrm{A}_{2}-\mathrm{H}_{2}$ reached a critical value (given in Table 2). The $\mathrm{A}_{2}-\mathrm{H}_{2}$ prolongation caused by a break phenomenon did not always reach this critical value.

In all cases of junctional tachycardia the $\mathbf{H A}^{\prime}$ intervals (from $\mathrm{H}$ to the start of the retrograde atrial depolarization) were shorter than the $A^{\prime} H$ intervals. During supraventricular tachycardia this $\mathrm{HA}^{\prime}$ interval remained constant and did not depend on the basic cycle length or the preceding $\mathrm{A}_{2}-\mathrm{H}_{2}$ interval.

In all patients who showed disappearance of the break phenomenon after atropine medication supraventricular tachycardia could not be provoked by a premature atrial depolarization (Cases 1, 5, 7, and 13). In the remaining patients, with the exception of Case 12, atrial echoes could be elicited after atropine by a premature atrial depolarization at a basic cycle length at which break phenomena could be demonstrated. However, in Case 12 after atropine the increment of the $\mathrm{A}_{2}-\mathrm{H}_{2}$ interval (AHmax. in Table 2) was less, so that the critical $\mathrm{A}_{2}-\mathrm{H}_{2}$ interval could not be reached.

In two patients (Cases 1 and 6) junctional tachycardias could be elicited only after orciprena- line medication. In Case 1 study at high rates after atropine could not be achieved for technical reasons. Therefore it cannot be excluded that the break phenomenon that occurred after orciprenaline medication might also be provoked at similar frequencies after atropine. The behaviour of Case 6 after administration of orciprenaline is presented in Fig. 5e and $f$ and in Figs. 6e and $f$. At a basic cycle length of $500 \mathrm{~ms}$ orciprenaline shortened the $A_{1}-H_{1}$ interval to $80 \mathrm{~ms}$ (Fig. 5e). A sudden prolongation of the $\mathrm{A}_{2}-\mathrm{H}_{2}$ interval from 110 to $190 \mathrm{~ms}$ could be provoked at a coupling interval of $270 \mathrm{~ms}$. The $\mathrm{A}_{2}-\mathrm{H}_{2}$ prolongation to $210 \mathrm{~ms}$ at a coupling interval of $240 \mathrm{~ms}$ (Fig. 5f) caused a stable supraventricular tachycardia, while under control conditions and after atropine medication the re-entry mechanism was spontaneously terminated after the two atrial echoes depicted. The critical $\mathrm{A}_{2}-\mathrm{H}_{2}$ interval was shortened from $320 \mathrm{~ms}$ (control) and $290 \mathrm{~ms}$ (after atropine) to $210 \mathrm{~ms}$.

\section{Discussion}

As suggested by Moe and Mendez (1966), longitudinal dissociation of the AV junction 'can be adequately explained on the basis of differences in effective refractory period without invoking basic differences in conduction velocity'. While the break phenomenon may represent substitution of a fast conducting pathway by a slow conducting pathway (Rosen et al., 1974), it could also be caused by a 
sudden increase in local conduction delay within a relatively refractory channel (Hoffman et al., 1963). However, the coincidence of the break phenomenon in human beings with the occurrence of echo phenomena and supraventricular tachycardia strongly points to the first hypothesis, which we will follow for the interpretation of our findings.

\section{Rate dependency of refractory periods and} the echo zone

Shortening of the cycle length led to a uniform decrease of the atrial effective refractory period in our patients, which agrees with the findings of Cagin et al. (1973), and Denes et al. (1974a). According to these authors prolongation of the effective refractory period of the AV node should be expected with cardiac acceleration. While this was the case in most of our patients, the refractory period of the slow pathway shortened in 1 patient and of the fast pathway in 2 patients.

The echo zone is defined by that critical time interval in which spontaneous or induced premature depolarizations can elicit echo phenomena. It is limited by the effective refractory period of the slow pathways or that of the atria on one side and on the other by the refractory period of the fast pathway or the $A_{1}-A_{2}$ interval at which the critical $A_{2}-H_{2}$ prolongation is reached. Rate-dependent changes of the refractory periods should therefore affect the echo zone. Taking into account these factors under control conditions and their different rate dependency, uniform behaviour of the echo zone should not be expected with cardiac acceleration. In accord with this, widening as well as narrowing of the echo zone was found when heart rate was increased.

\section{Influence of the autonomic nervous system on the refractory periods}

Owing to its anticholinergic action atropine invariably shortens the effective refractory period of the slow and fast conducting pathways. Improvement in antegrade as well as retrograde $\mathrm{AV}$ nodal conduction encourages the occurrence of atrial echoes (Case 7) and the induction of stable junctional tachycardias (Case 14). After atropine medication the break phenomenon can be elicited with greater or less ease depending on the basic conditions. With a relatively short effective refractory period of the fast pathway further shortening by atropine can cause disappearance of the break phenomenon (Fig. 4). If the fast-conducting pathway is antegradely blocked under control conditions, shortening of its effective refractory period by atropine may cause the appearance of the break phenomenon at the identical basic cycle length (Case 6, Fig. 6). Furthermore, the echo zone can be influenced by shortening of the critical $\mathrm{A}_{2}-\mathrm{H}_{2}$ interval (Case 12). This may be explained by the decrease of the effective refractory period of an upper common pathway in the re-entry circuit (Mendez et al., 1965).

As expected, the effect of orciprenaline is similar to that of atropine. These findings clearly demonstrate the importance of changes in sympathetic and/ or parasympathetic nervous tone in relation to the trigger mechanism of junctional tachycardias. It emphasizes the role of the autonomic imbalance often found in patients prone to paroxysmal supraventricular tachycardia.

\section{Anatomical substrate of dual AV conduction}

It has previously been suggested that dual AV conduction is anatomically correlated with a paranodal bypass or with longitudinal dissociation of the AV node (Kistin, 1965). The specific pharmacological influence of atropine on the AV node (Akhtar et al., 1974) suggests that the AV node is included in the fast-conducting pathway. This points to a longitudinal dissociation of the AV node. However, if a paranodal bypass inserts into the distal part of the AV node, the shortening of the effective refractory period of the fast-conducting pathway could be caused by the behaviour of the refractory period of the nodal part of this pathway.

Usually the effective refractory period of the AV node increases whereas the refractory periods of the atria and bundle of His decreases when the basic cycle length is shortened (Denes et al., 1974a; Cagin et al., 1973). The prolongation of the effective refractory period of the fast pathway with cardiae acceleration in some patients suggests-in the light of the considerations mentioned above-a longitudinal dissociation. On the other hand, the shortening of the refractory period of the fast pathway with decreasing basic cycle length in other patients could be explained by a paranodal bypass system.

Further pharmacological investigations may well be helpful to determine the anatomical substrate in these patients (Spurrell, Krikler, and Sowton, 1974).

\section{Occurrence of break phenomenon and provocation of junctional tachycardias}

As previously shown, a critical combination of the effective refractory periods of the atria, or the upper common pathway, and the fast- and the slowconducting pathways is needed for the manifestation of both these phenomena. From a consideration of the individual properties of these refractory periods under control conditions, one would expect that 


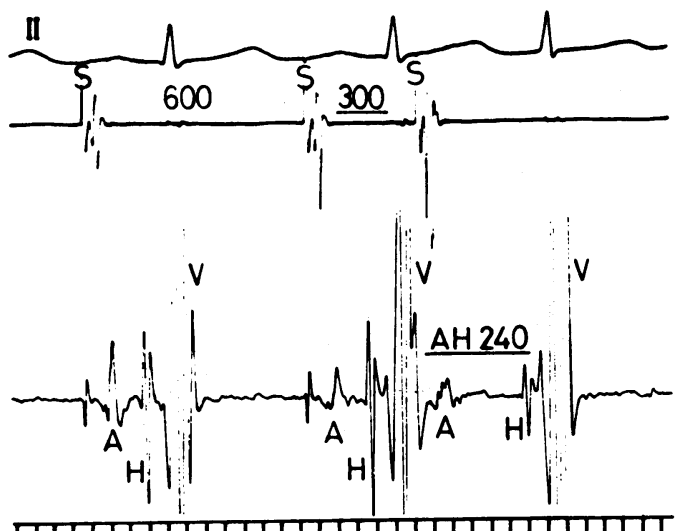

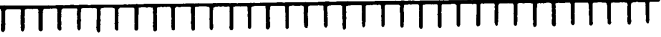
a)
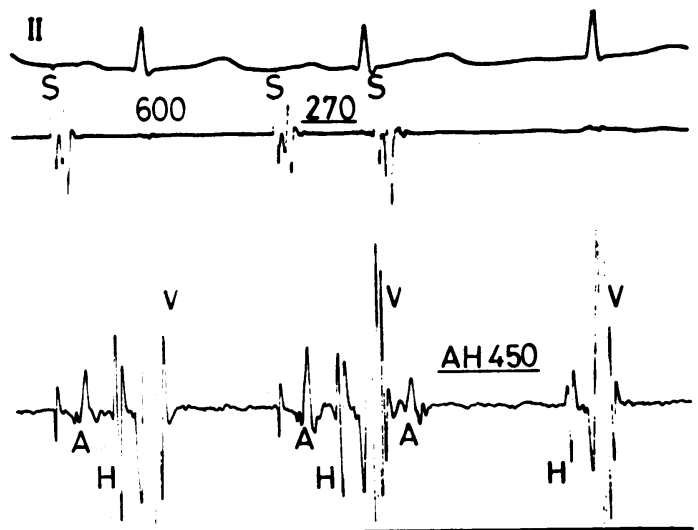

b) IIIIIIIIIIIIIIITाIIIIIIIT

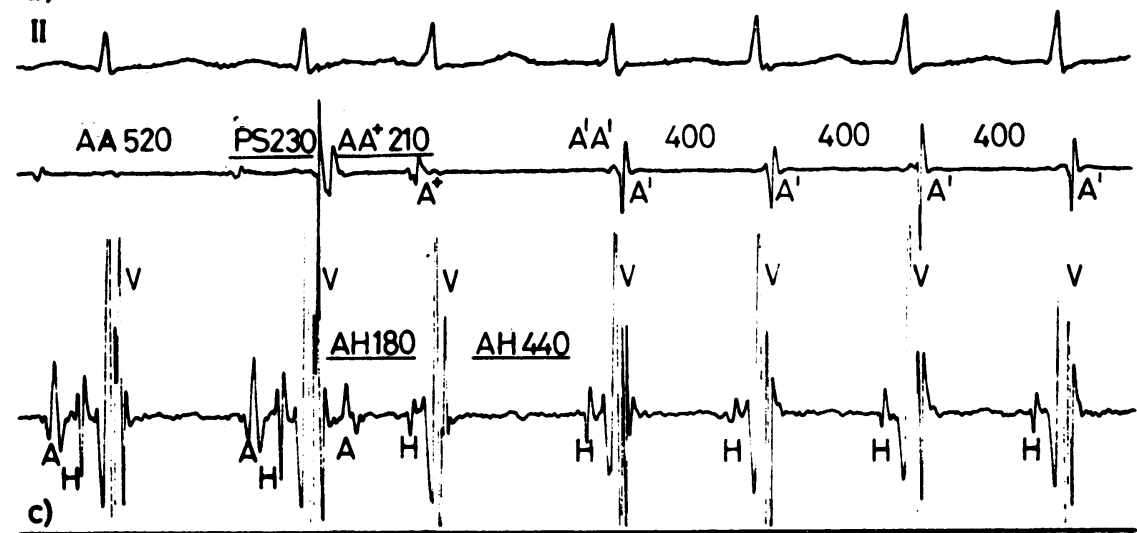

FIG. 7 Electrocardiogram (lead II), atrial electrogram, and His bundle recording in Case 7. Panel $a$ and $b=$ control conditions. (a) Basic cycle length $600 \mathrm{~ms}$ : at an $S_{1}-S_{2}$ interval of $300 \mathrm{~ms} \mathrm{~A}_{2}-\mathrm{H}_{2}$ measured $240 \mathrm{~ms}$. (b) Basic cycle length $600 \mathrm{~ms}$ : shortening of $\mathrm{S}_{1}-\mathrm{S}_{2}$ to $270 \mathrm{~ms}$ resulted in a prolongation of $A_{2}-H_{2}$ to $450 \mathrm{~ms}$. (c) After atropine medication: at sinus rhythm with a cycle length of $520 \mathrm{~ms}$ a premature atrial depolarization with a coupling interval of $230 \mathrm{~ms}$ was conducted with an $\mathrm{A}_{2}-\mathrm{H}_{2}$ interval of $180 \mathrm{~ms}$. A short-coupled atrial extrasystole $\left(A^{+}\right)$, after this induced premature atrial depolarization produced a similar $A H$ prolongation as in panel $b$ and was followed by a junctional tachycardia.

these phenomena might be provoked by changing basic cycle length (Cases 2, 5, and 7) or by pharmacological intervention (Case 6). The break phenomenon may be considered as a diagnostic clue for the presence of dual AV conduction; patients with paroxysmal supraventricular tachycardia should be studied by the provocative methods described to elicit it.

The association of the break phenomenon with the occurrence of atrial echoes can be explained by the development of a unidirectional block of the fast-conducting pathway. However, it is possible that such a unidirectional block could already exist in sinus rhythm when the fast pathway would be concealed. Thereby atrial echoes could occur without a previous break phenomenon at a critical $A_{2}-H_{2}$ prolongation. This interpretation is favoured by the provocation of a break phenomenon by the positive dromotropic effect of the pharmacological agents used at the same basic cycle length (Fig. 5 and 6).

On the other hand, it is necessary to have sufficient delay in the re-entry cycle to be able to provoke junctional tachycardias, otherwise the structures 
previously depolarized (upper common pathway) cannot be activated again. So, in spite of a break phenomenon and a significant $\mathrm{A}_{2}-\mathrm{H}_{2}$ prolongation, refractoriness of the upper common pathway may prevent atrial echoes. Shortening of this refractory period by pharmacological agents or by changing the basic cycle length may therefore permit atrial echoes.

In Case 7 at a basic stimulated cycle length of $600 \mathrm{~ms}$ and a coupling interval of $270 \mathrm{~ms}$ a break phenomenon (increment 240 to $450 \mathrm{~ms}$ ) occurred. Atrial echoes failed to appear (Fig. 7a and b). After atropine the spontaneous basic cycle length was $520 \mathrm{~ms}$. Premature atrial depolarizations were not able to provoke a break phenomenon. An additional short-coupled atrial extrasystole after an induced premature atrial depolarization produced a similar $\mathrm{AH}$ prolongation, as in Fig. $7 \mathrm{~b}$, and was followed by a junctional tachycardia (Fig. 7c). A possible explanation is offered: Atropine shortened the effective refractory period of the fast pathway in such a way that a unidirectional block could be provoked only by a dual premature atrial depolarization; shortening of the refractory period of the upper common pathway now allowed the occurrence of atrial echoes at AH intervals which did not permit this before atropine administration.

The phenomena described depend on a critical combination of conduction times and refractory periods of the structures between atria and the bundle of His which were shown to be very sensitive to changes in rate and to the administration of pharmacological agents affecting the autonomic nervous control of the heart. Because of these complex multifactorial interrelations a wide inter-individual variability can be observed in the various factors involved.

\section{References}

Akhtar, M., Damato, A. N., Caracta, A. R., Batsford, W. P., Josephson, M. E., and Lau, S. H. (1974). Electrophysiological effects of atropine on atrioventricular conduction studied by His bundle electrograms. American fournal of Cardiology, 33, 333.

Cagin, N. A., Kunstadt, D., Wolfish, P., and Levitt, B. (1973). The influence of heart rate on the refractory period of the atrium and the A-V conducting system. American Heart fournal, 85, 358.

Denes, P., Wu, D., Dhingra, R. C., Chuquimia, R., and Rosen, K. M. (1973). Demonstration of dual A-V nodal pathways in patients with paroxysmal supraventricular tachycardia. Circulation, 48, 549.

Denes, P., Wu, D., Dhingra, R. C., Pietras, R. J., and Rosen, K. M. (1974a). The effects of cycle length on cardiac refractory periods in man. Circulation, 49, 32.

Denes, P., Wu, D., and Rosen, K. M. (1974b). Demonstration of dual $\mathrm{A}-\mathrm{V}$ pathways in a patient with Lown-GanongLevine syndrome. Chest, 65, 343.

Goldreyer, B. N., and Bigger, J. T. (1971). Site of re-entry in paroxysmal supraventricular tachycardia in man. Circulation, 43, 15.

Goldreyer, B. N., Weiss, M. B., and Damato, A. N. (1971). Supraventricular tachycardia initiated by sinus beats. Circulation, 44, 820.

Hoffman, B. F., Moore, E. N., Stuckey, J. H., and Cranefield, P. F. (1963). Functional properties of the atrioventricular conduction system. Circulation Research, 13, 308.

Janse, M. J., Capelle, F. J. L. van, Freud, G. E., and Durrer, D. (1971). Circus movement within the AV node as a basis for supraventricular tachycardia as shown by multiple micro-electrode recording in the isolated rabbit heart. Circulation Research, 28, 403.

Kistin, A. D. (1965). Atrial reciprocal rhythm. Circulation, 32, 687.

Mendez, C., Han, J., Jalon, P. D. G. de, and Moe, G. K. (1965). Some characteristics of ventricular echoes. Circulation Research, 16, 562.

Moe, G. K., Cohen, W., and Vick, R. L. (1963). Experimentally induced paroxysmal A-V nodal tachycardia in the dog. American Heart Fournal, 65, 87.

Moe, G. K., and Mendez, C. (1966). The physiological basis of reciprocal rhythm. Progress in Cardiovascular Diseases, $8,461$.

Neuss, H., and Schlepper, M. (1974). Unusual re-entry mechanisms in patients with Wolff-Parkinson-White syndrome. British Heart fournal, 36, 880.

Rosen, K. M., Mehta, A., and Miller, R. A. (1974). Demonstration of dual atrioventricular nodal pathways in man. American fournal of Cardiology, 33, 291.

Scherlag, B. J., Lau, S. H., Helfant, R. H., Berkowitz, W. D., Stein, E., and Damato, A. N. (1969). Catheter technique for recording His bundle activity in man. Circulation, 39, 13.

Spurrell, R. A. J., Krikler, D. M., and Sowton, E. (1974). Concealed bypasses of the atrioventricular node in patients with paroxysmal supraventricular tachycardia revealed by intracardiac electrical stimulation and Verapamil. American fournal of Cardiology, 33, 590.

Van Capelle, F. J. L., Janse, M. J., Varghese, P. J., Freud, G. E., Mater, C., and Durrer, D. (1972). Spread of excitation in the atrioventricular node of isolated rabbit hearts studied by multiple microelectrode recording. Circulation Research, 31, 602.

Wit, A. L., Weiss, M. B., Berkowitz, W. D., Rosen, K. M., Steiner, C., and Damato, A. N. (1970). Patterns of atrioventricular conduction in the human heart. Circulation Research, 2, 345.

Requests for reprints to Professor M. Schlepper, Kerckhoff-Klinik, 635 Bad Nauheim, Benekestrasse 6-8, West Germany. 